Check for updates

Cite this: RSC Adv., 2020, 10, 27575

\section{Stöber synthesis of salen-formaldehyde resin polymer- and carbon spheres with high nitrogen content and application of the corresponding $\mathrm{Mn}$ - containing carbon spheres as efficient electrocatalysts for the oxygen reduction reaction $\uparrow$}

\author{
Tahereh Hosseinzadeh Sanatkar, ${ }^{a}$ Alireza Khorshidi, (DD *a Rouhollah Yaghoubi, ${ }^{\text {b }}$ \\ Esmail Sohouli ${ }^{c}$ and Jamaladin Shakeri ${ }^{d}$
}

\begin{abstract}
Salen-formaldehyde (SF) resin polymer spheres were synthesized by the Stöber method from 4,4'dihydroxysalen ( $N, N^{\prime}$-bis-(4-hydroxysalicylidene)-ethylenediamine; a tetradentate $\mathrm{N}_{2} \mathrm{O}_{2}$ Schiff base ligand) and formaldehyde. The salen precursor was prepared by condensation of ethylenediamine with 2,4-dihydroxybenzaldehyde in methanol. The SF resin colloidal spheres were also prepared by using Pluronic F127 and ammonia as a porogenic agent and catalyst, respectively (SF-P). In addition, corresponding $\mathrm{Mn}(॥)$-coordinated polymer spheres of the SF-P were synthesized (SF-P-Mn(॥)). Corresponding monodispersed carbon spheres of all of the abovementioned samples were also obtained by pyrolysis technique. All of the products were characterized with conventional microscopic and spectroscopic techniques, as well as other physical methods such as BET analysis. It was found that carbonization of the SF resin spheres results in carbon spheres with specific surface areas in the range of 499-528 $\mathrm{m}^{2} \mathrm{~g}^{-1}$ and average pore sizes in the range of 2.58-3.08 $\mathrm{nm}$. Nitrogen content of the SF$\mathrm{MW}_{\mathrm{HT}}$ (obtained hydrothermally in a methanol/water mixture), and SF-P-C@Mn (obtained from carbonization of SF-P-Mn(॥)) samples were as high as 27.5 wt\% and 35.02 wt\%, respectively. Finally, a glassy carbon electrode (GCE) modified with SF-P-CaMn (SF-P-CaMn/GCE) was prepared and its electrocatalytic activity was evaluated for oxygen reduction reaction (ORR) by linear sweep voltammetry (LSV). The LSV results showed that the SF-P-CaMn/GCE has a higher current density and a lower negative potential in the ORR compared to GCE.
\end{abstract}

Received 28th April 2020 Accepted 7th July 2020

DOI: $10.1039 / \mathrm{dOra03815f}$

rsc.li/rsc-advances features. ${ }^{7}$ Various strategies have been reported for the preparation of polymer colloids and their derived carbon-based nanomaterials. ${ }^{8-10}$

Carbon nanospheres have been synthesized by several methods such as chemical vapor deposition (CVD), nanocasting with silica spheres as hard templates, hydrothermal carbonization of sugars, Stöber synthesis, soft-templating strategies via organic-organic self-assembly, etc. ${ }^{\mathbf{1 1}}$ The well-known Stöber method was first reported in 1968 for the synthesis of colloidal silica spheres via controlled hydrolysis and condensation of Sicontaining compounds such as tetraethyl orthosilicate (TEOS) with ammonia as a catalyst in aqueous alcoholic solution. ${ }^{\mathbf{1 2}}$ Later, Liu et al. reported a modification to the Stöber synthesis for preparation of resorcinol formaldehyde (RF) polymer spheres with uniform and controllable particle size on submicrometer scale, and subsequent conversion of these spheres into carbon spheres via pyrolysis. ${ }^{13}$ Particle size of the resultant polymer- or carbon spheres can be controlled by varying the experimental parameters, including the alkyl chain of the alcohol, alcohol/water ratio, the amount of ammonia, 
formaldehyde, RF precursor, and so on. ${ }^{13}$ Resorcinol formaldehyde (RF) resins are interesting precursors which can be used to prepare highly porous carbon materials. ${ }^{\mathbf{1 4 , 1 5}}$ On the other hand, various polymeric precursors, such as glucose, ${ }^{16}$ dopamine, ${ }^{17}$ tannic acid, ${ }^{18}$ phenol-formaldehyde resin ${ }^{19}$ and conducting polymers ${ }^{20}$ etc., have been successfully used to synthesize new uniform nanospheres, and provided new opportunities in the research area of colloidal and carbon spheres. The scope of applications of carbon materials have been made even more extensive by heteroatom-doping. ${ }^{21,22}$ Doping heteroatoms such as nitrogen, sulfur and phosphorus, even in small quantities in the carbon framework, can lead to significant enhancements in the performance of polymer colloids and their derived carbon-based nanomaterials. ${ }^{23,24}$ Nitrogen is the most widely used heteroatom that can be doped directly into the polymer colloids.

Schiff bases are a group of compounds that contain nitrogen atoms in their structure. They are characterized by an imine or azomethine group $(-\mathrm{RC}=\mathrm{N}-$ ), and are generally synthesized by condensation of primary amines with carbonyl compounds. ${ }^{25}$ They form an important class of ligands and have gained considerable attention due to their diverse applications such as catalysis, ${ }^{26}$ electrochemistry, ${ }^{27}$ bioscience,${ }^{28}$ optics,${ }^{29}$ host-guest chemistry $^{30}$ and molecular recognition. ${ }^{31}$ Moreover, Schiff bases have been successfully used to remove heavy metals selectively from aqueous media. ${ }^{32}$ Among Schiff bases, salentype compounds are an interesting class of the tetradentate ligands with four donor sites $\left(\mathrm{N}_{2} \mathrm{O}_{2}\right) \cdot{ }^{33}$ As shown in Scheme 1, the 4,4'-dihydroxysalen ligand provides an ideal environment for equatorial coordination of various transition metals. While synthesis of polymer spheres with high nitrogen content is highly desirable for specific applications, there are few reports on preparation of such monodisperse polymer spheres. ${ }^{22,34}$

Carbon nanospheres have been recognized as promising electrocatalysts for the oxygen reduction reaction (ORR) ${ }^{13} \mathrm{~N}$ doped mesoporous carbon nanospheres exhibit unique features, including enhanced dissociation of $\mathrm{O}_{2}$ within the pores, and improved wettability and charge/spin density on the carbon, to act as an electrode for the ORR. ${ }^{35}$ Moreover, decoration of the carbon nanospheres with electroactive metal nanoparticles can obviously enhance the ORR capacity. ${ }^{\mathbf{1 8}}$

Herein, we report the synthesis of monodisperse salenformaldehyde (SF) resin spheres by Stöber method using 4,4'dihydroxysalen and formaldehyde as precursors, and Pluronic F127 as a template and porogenic agent. In addition, the corresponding polymeric metal complex spheres of the SF resin were obtained by deprotonation of the tetradentate $\left(\mathrm{N}_{2} \mathrm{O}_{2}\right)$ salen moieties and coordination with $\mathrm{Mn}$ (II) by taking advantage of

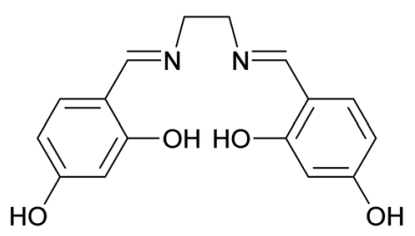

Scheme 1 Molecular structure of 4,4'-dihydroxysalen. the chelating effect. Moreover, pyrolysis technique was used to prepare carbon spheres of the aforementioned resin polymers. Finally, Mn nanoparticles-loaded carbon spheres were successfully used as an electrocatalyst for the ORR process.

\section{Experimental}

\section{Materials and methods}

All of the chemicals including 2,4-dihydroxybenzaldehyde and ethylenediamine were purchased from Merck (http:// www.merck.com) or Sigma-Aldrich (http:// www.sigmaaldrich.com) Companies.

All solvents and reagents were of analytical grade and used without further purification. A Heraeus CHN-O-Rapid elemental analyzer was used for elemental analyses. FT-IR spectra were recorded on a Bruker Alpha spectrophotometer using $\mathrm{KBr}$ pellets over the range of $4000-400 \mathrm{~cm}^{-1}$. UV-Vis spectra were recorded on a Rayleigh UV-1800 spectrophotometer. ${ }^{1} \mathrm{H}$ NMR spectra were recorded in DMSO- $d_{6}$ on a Bruker Avance III $300 \mathrm{MHz}$ spectrometer at $298 \mathrm{~K}$ using tetramethylsilane as an internal standard. X-ray powder diffraction (XRD) data were acquired on a Philips PW1730 diffractometer with $\mathrm{Cu} \mathrm{K} \alpha$ radiation $(\lambda=1.5406 \AA)$. Field emission scanning electron microscopy (FESEM) images of the synthesized samples were obtained using a Tescan Mira III scanning electron microscope (Tescan, Czech Republic). A layer of Au was used for all samples before imaging. Energy dispersive X-ray technique (EDX) was used for surface elemental analysis. TEM imaging was performed on a Zeiss-EM10C microscope at an accelerating voltage of $100 \mathrm{kV}$. Brunauer-Emmett-Teller (BET) analyses were performed at $77 \mathrm{~K}$ on a Belsorp mini II gas adsorption analyzer, Japan. All of the samples were degassed at $200{ }^{\circ} \mathrm{C}$ for $2 \mathrm{~h}$ before BET analysis. X-ray photoelectron spectroscopy (XPS) analysis was performed by Gamma data-scienta ESCA 200 hemispherical analyzer equipped with an $\mathrm{Al} \mathrm{K} \alpha$ (1486.6 eV) X-ray source.

\section{Electrochemical measurements}

All of the electrochemical measurements were carried out on a potentiostat/galvanostat of the type Autolab PGSTAT302N (Eco Chemie B. V., Switzerland) driven by the Nova 2.1.2 software. A conventional three-electrode setup including a modified or unmodified GCE working electrode, an $\mathrm{Ag} / \mathrm{AgCl}(3.00 \mathrm{M} \mathrm{KCl})$ reference electrode, and a Pt wire auxiliary electrode (Azar Electrode, Iran) was used. Some electrochemical techniques such as cyclic voltammetry (CV), electrochemical impedance spectroscopy (EIS) and amperometry were used in the experiments. The EIS measurements were performed at a frequency range of $10.0 \mathrm{kHz}$ to $10 \mathrm{MHz}$, and $0.1 \mathrm{M} \mathrm{KOH}$ was utilized as a redox probe.

\section{Syntheses}

\section{Synthesis of the salen compound}

The $\quad 4,4^{\prime}$-dihydroxysalen $\quad\left(N, N^{\prime}\right.$-bis-(4-hydroxysalicylidene)ethylenediamine) was synthesized by condensation of 
ethylenediamine with 2,4-dihydroxybenzaldehyde in methanol. Briefly, ethylenediamine $(0.33 \mathrm{~mL}, 5.0 \mathrm{mmol})$ was added dropwise to a solution of 2,4-dihydroxybenzaldehyde (1.38 g, 10.0 $\mathrm{mmol}$ ) in $100 \mathrm{~mL}$ of methanol at room temperature. The reaction mixture was then refluxed for $3 \mathrm{~h}$ with continuous stirring. After cooling down to room temperature, a yellow precipitate was filtered off and washed with methanol. Yield: $88 \%$. Anal. calcd for $\mathrm{C}_{16} \mathrm{H}_{16} \mathrm{~N}_{2} \mathrm{O}_{4}\left(\mathrm{MW}=300.31 \mathrm{~g} \mathrm{~mol}^{-1}\right)$ : C, 63.99; H, 5.37; N, 9.33. Found: C, 63.87; H, 5.45; N, 9.41\%. IR (KBr pellet, $\left.\mathrm{cm}^{-1}\right): 3416$ (s, $\left.\nu_{\mathrm{OH}}\right), 1639\left(\mathrm{~s}, \nu_{\mathrm{C}=\mathrm{N}}\right), 1234\left(\mathrm{~m}, \nu_{\mathrm{Ph}-\mathrm{O}}\right) . \quad$ UV-Vis: $\lambda_{\max }(\mathrm{nm})$ ( $\varepsilon, \mathrm{M}^{-1} \mathrm{~cm}^{-1}$ ) (DMSO): 280 (25 750), 308 (21 100), 390 (2100). ${ }^{1} \mathrm{H}$ NMR (DMSO- $\left.d_{6}, 300 \mathrm{MHz}, \mathrm{ppm}\right) ; \delta: 13.58$ (br, $2 \mathrm{H}^{7}$ ), 9.80 (br, $\left.2 \mathrm{H}^{5}\right), 8.37\left(\mathrm{~s}, 2 \mathrm{H}^{2}\right), 7.18\left(\mathrm{~d}, 2 \mathrm{H}^{3}, J=8.4 \mathrm{~Hz}\right), 6.28\left(\mathrm{dd}, 2 \mathrm{H}^{4}, J=8.4\right.$ and $2.4 \mathrm{~Hz}), 6.27\left(\mathrm{~d}, 2 \mathrm{H}^{6}, J=2.4 \mathrm{~Hz}\right), 3.79\left(\mathrm{~s}, 4 \mathrm{H}^{1}\right)$.

\section{Synthesis of the SF resin polymer spheres}

Monodisperse SF resin spheres were synthesized by using 4,4'dihydroxysalen and formaldehyde as precursors. In a typical synthesis, ammonia solution ( $25 \mathrm{wt} \%, 0.1 \mathrm{~mL}$ ) was mixed with $16 \mathrm{~mL}$ of absolute methanol and $12 \mathrm{~mL}$ of deionized water and stirred for $1 \mathrm{~h}$ at $30{ }^{\circ} \mathrm{C}$. Then, $4,4^{\prime}$-dihydroxysalen $(0.55 \mathrm{~g})$ was added and magnetically stirred for $30 \mathrm{~min}$. Afterwards, $0.28 \mathrm{~mL}$ of $37 \%$ formaldehyde solution was added dropwise to the above solution. After few minutes, the solution became cloudy and stirring was continued for $24 \mathrm{~h}$ at the same temperature. A lightyellow solid product was obtained after centrifugation followed by air-drying at $100{ }^{\circ} \mathrm{C}$, which was denoted as SF-MW (73\% yield).

The same procedure was repeated and right before separation of the product, a hydrothermal process was applied in a Teflonlined autoclave at $100{ }^{\circ} \mathrm{C}$ for $24 \mathrm{~h}$ under a static condition. After cooling down to room temperature, the dark-yellow SF resin polymer spheres were separated by centrifugation and air-dried at $100{ }^{\circ} \mathrm{C}$ for $24 \mathrm{~h}$. The product was denoted as SF-MW $\mathrm{HT}_{\mathrm{HT}}(70 \%$ yield).

In addition, hydrothermally processed SF resin polymer spheres were obtained in the same manner in $28 \mathrm{~mL}$ of absolute methanol (instead of a $16: 12$ mixture of $\mathrm{MeOH}: \mathrm{H}_{2} \mathrm{O}$ mixture), which were denoted as $\mathrm{SF}-\mathrm{M}_{\mathrm{HT}}(76 \%$ yield).

\section{Pluronic F127-directed synthesis of the SF resin polymer spheres (SF-P)}

The SF-P spheres were synthesized by the abovementioned precursors and triblock copolymer Pluronic F127. The overall

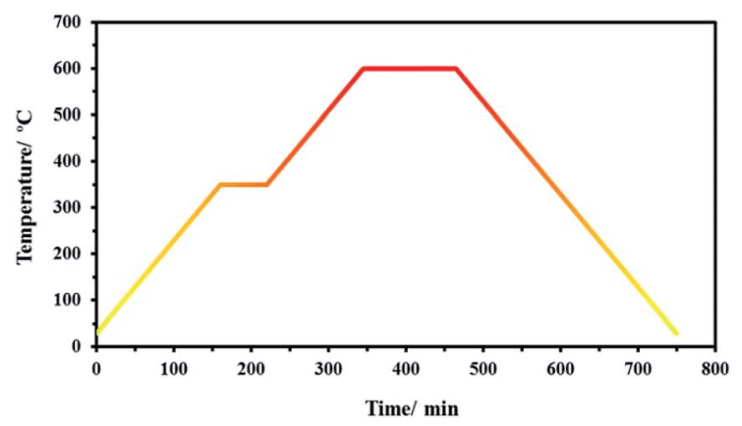

Fig. 1 The heating program used for carbonization. process was similar to that described above section. First, ammonia solution ( $25 \mathrm{wt} \%, 0.1 \mathrm{~mL}$ ) was mixed with $16 \mathrm{~mL}$ of absolute methanol and $12 \mathrm{~mL}$ of deionized water and stirred for $1 \mathrm{~h}$ at $30{ }^{\circ} \mathrm{C}$. Then, 4,4'-dihydroxysalen ( $\left.0.55 \mathrm{~g}\right)$ was added and magnetically stirred for $30 \mathrm{~min}$. Afterwards, $0.27 \mathrm{~g}$ of triblock copolymer Pluronic F127 was added and stirred for $30 \mathrm{~min}$. In the next step, $0.28 \mathrm{~mL}$ of $37 \%$ formaldehyde solution was added dropwise. After few minutes, the solution became cloudy and stirring was continued for $24 \mathrm{~h}$ at the same temperature. The light-yellow reaction mixture was then transferred into a Teflonlined autoclave and heated at $100{ }^{\circ} \mathrm{C}$ for $24 \mathrm{~h}$. After cooling down to room temperature, a dark-yellow solid product was obtained after centrifugation followed by air-drying at $100{ }^{\circ} \mathrm{C}$, which was denoted as SF-P (68\% yield).

\section{Synthesis of the Mn(II)-coordinated SF-P spheres (SF-P-Mn(II))}

The overall process for the synthesis of SF-P-Mn(II) was similar to that described in above sections. First, ammonia solution (25 wt\%, $0.1 \mathrm{~mL}$ ) was mixed with $16 \mathrm{~mL}$ of absolute methanol and $12 \mathrm{~mL}$ of deionized water and stirred for $1 \mathrm{~h}$ at $30^{\circ} \mathrm{C}$. Then, 4,4'-dihydroxysalen ( $0.55 \mathrm{~g}$ ) was added and magnetically stirred for $30 \mathrm{~min}$. Afterwards, $0.27 \mathrm{~g}$ of triblock copolymer Pluronic F127 was added and stirred for $30 \mathrm{~min}$. In the next step, $0.28 \mathrm{~mL}$ of $37 \%$ formaldehyde solution was added dropwise. After few minutes, the solution became cloudy and stirring was continued for $24 \mathrm{~h}$ at the same temperature. Then a methanolic solution of $\mathrm{Mn}\left(\mathrm{NO}_{3}\right)_{2} \cdot 4 \mathrm{H}_{2} \mathrm{O}(2 \mathrm{~mL}, 0.1 \mathrm{M})$ was added dropwise to the reaction mixture and stirred for $8 \mathrm{~h}$. It should be mentioned that after addition of the Mn(II) salt, color of the solution became dark red. Then the reaction mixture was transferred into a Teflon-lined autoclave and heated at $100{ }^{\circ} \mathrm{C}$ for $24 \mathrm{~h}$. After cooling down to room temperature, the polymeric $\mathrm{Mn}$ (II) complex was collected by centrifugation and dried at $100{ }^{\circ} \mathrm{C}$ for $24 \mathrm{~h}$ (67\% yield).

\section{Carbonization of the SF resin polymer spheres}

For carbonization, the precursors were transferred in a quartz boat into a tube furnace and heated under $\mathrm{N}_{2}$ atmosphere. As



Fig. 2 FT-IR spectra of (a) 4,4' -dihydroxysalen, (b) SF-MW ${ }_{H T}$, (c) SF-P$C$, and (d) SF-P-CaMn. 


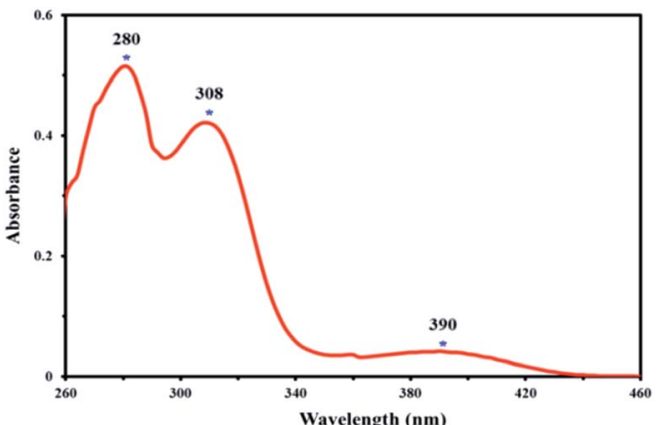

Fig. 3 UV-Vis spectrum of $4,4^{\prime}$-dihydroxysalen in DMSO $\left(2 \times 10^{-5} \mathrm{M}\right)$ at room temperature.



Fig. $4{ }^{1} \mathrm{H}$ NMR spectrum of $4,4^{\prime}$-dihydroxysalen in DMSO- $d_{6}$.

shown in Fig. 1, first, the temperature was raised from room temperature to $350{ }^{\circ} \mathrm{C}$ with a heating rate of $5{ }^{\circ} \mathrm{C} \min ^{-1}$ and kept at $350^{\circ} \mathrm{C}$ for $2 \mathrm{~h}$. Next, the temperature was raised to $600^{\circ} \mathrm{C}$ with a $5{ }^{\circ} \mathrm{C} \mathrm{min}{ }^{-1}$ heating rate and maintained at $600{ }^{\circ} \mathrm{C}$ for $4 \mathrm{~h}$. Finally, the samples were cooled down to room temperature. The obtained samples from SF-MW ${ }_{\mathrm{HT}}$, SF-P, and SF-P-Mn(II) were denoted as SF-C, SF-P-C, and SF-P-C@Mn, respectively.

\section{Preparation of the modified carbon paste electrode}

To prepare the SF-P-C@Mn-modified glassy carbon electrode, surface of the electrode was polished by alumina powder, washed with water, and sonicated in deionized water to eliminate any surface contamination. Then, $1.0 \mathrm{mg}$ of the SF-PC@Mn was mixed with $1.0 \mathrm{~mL}$ of water in a test tube and irradiated in an ultrasonic bath for $1 \mathrm{~h}$. Then, $5 \mu \mathrm{L}$ of this mixture was placed on the electrode in an oven at $50{ }^{\circ} \mathrm{C}$ to prepare the SF-P-C@Mn-modified glassy carbon electrode which was denoted as SF-P-C@Mn/GCE. The SF-C/GCE and SF-P-C/GCE were prepared in the same way.

\section{Results and discussion}

\section{Spectral characterization of the salen compound}

In the FT-IR spectrum of 4,4'-dihydroxysalen (Fig. 2a), broad band of the phenolic $\mathrm{O}-\mathrm{H}$ stretching $\left(\nu_{\mathrm{O}-\mathrm{H}}\right)$ was appeared at $3416 \mathrm{~cm}^{-1}$.

Strong band of the azomethine moiety $\left(\nu_{\mathrm{C}=\mathrm{N}}\right)$ was observed at $1639 \mathrm{~cm}^{-1}$, and that of the $\mathrm{C}-\mathrm{O}$ stretching $\left(\nu_{\mathrm{Ph}-\mathrm{O}}\right)$ was observed at $1234 \mathrm{~cm}^{-1}$.

Electronic absorption spectrum of the 4,4'-dihydroxysalen was recorded at room temperature in DMSO $\left(2 \times 10^{-5} \mathrm{M}\right)$. As shown in Fig. 3, the UV-Vis spectrum of 4,4'-dihydroxysalen has three absorption bands at 280, 308 and $390 \mathrm{~nm}$. The highly intense bands at 280 and $308 \mathrm{~nm}$ are assigned to $\pi \rightarrow \pi^{*}$ and $\mathrm{n}$ $\rightarrow \pi^{*}$ transitions of the salen, and the broad band at about $390 \mathrm{~nm}$ is attributed to intraligand charge-transfer. ${ }^{36}$

${ }^{1} \mathrm{H}$ NMR spectra of the salen Schiff base showed broad signals for phenolic $\mathrm{OH}^{7}$ and $\mathrm{OH}^{5}$ moieties at 13.58 and 9.80 ppm, respectively (Fig. 4). The aromatic protons resonated

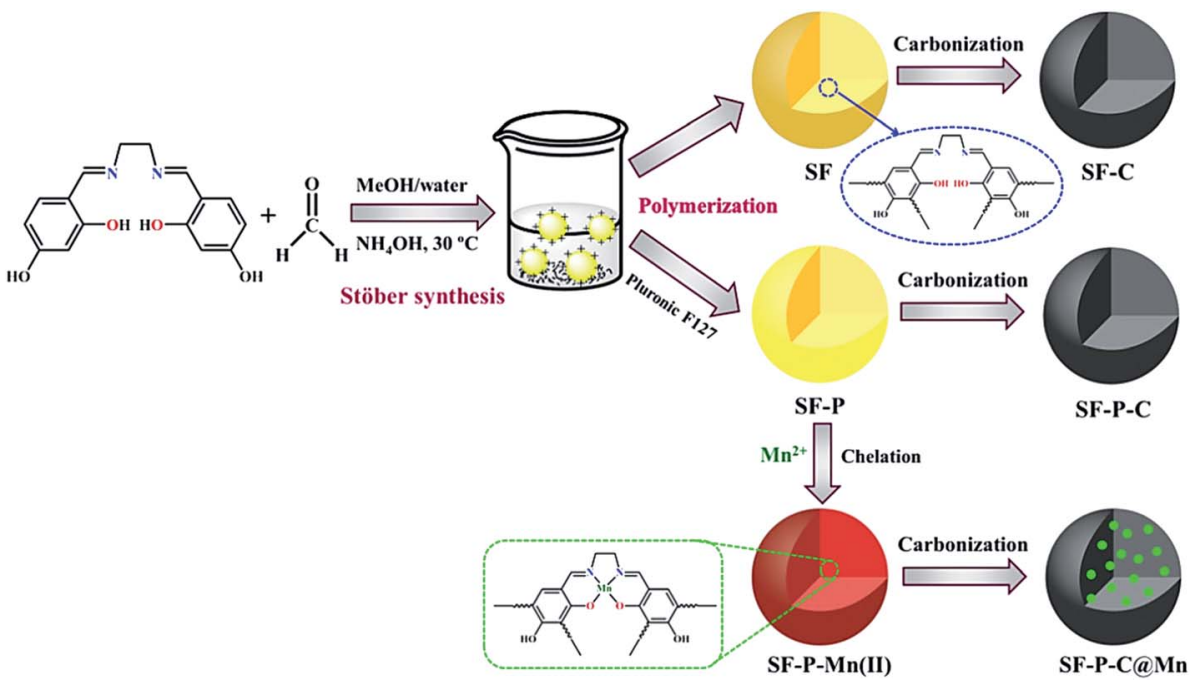

Fig. 5 A schematic illustration for preparation of the SF, SF-P and SF-P-Mn(॥) samples and their corresponding carbonization products. 

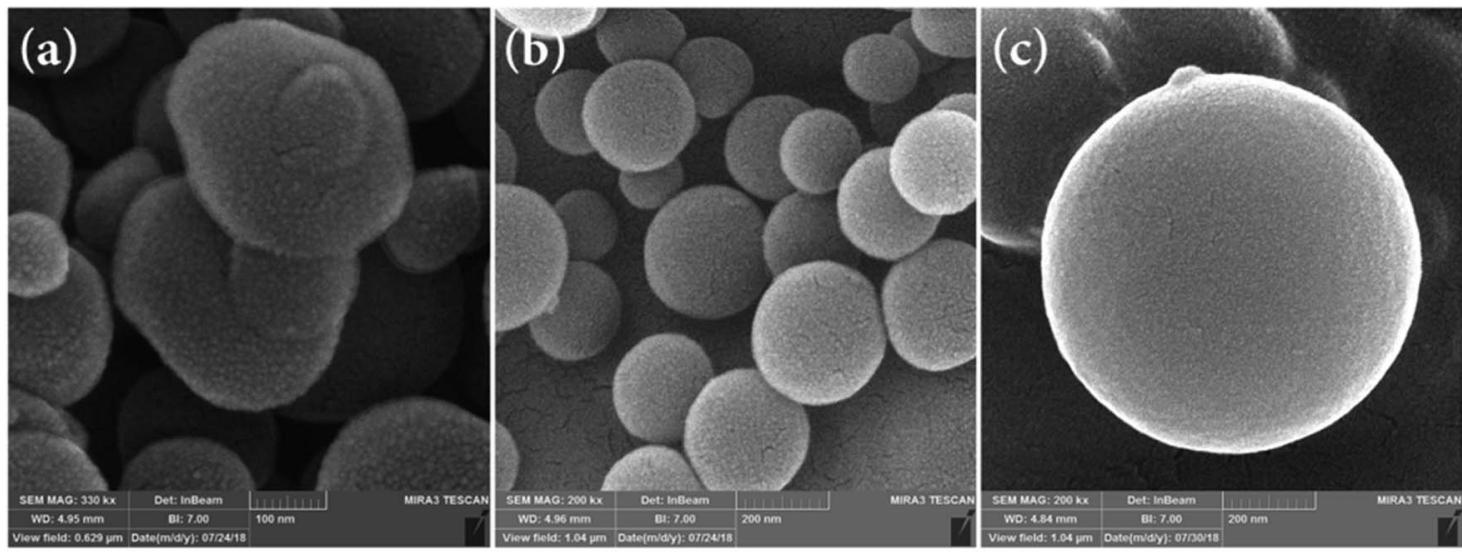

Fig. 6 SEM images of (a) SF-MW, (b) SF-MW ${ }_{H T}$, and (c) SF-M $M_{H T}$ resin polymer spheres.

in the range of 6.18-7.18 ppm and unambiguously assigned according to their coupling constants (see Experimental). The expected singlet for the proton of the imine moiety $\left(\mathrm{H}^{2}\right)$ was observed at $8.37 \mathrm{ppm}$, and the aliphatic protons $\left(\mathrm{H}^{1}\right)$ were observed at $3.79 \mathrm{ppm}$ as a singlet.

\section{Characterization of the prepared resin polymer- and carbon spheres}

A schematic illustration for preparation of the SF, SF-P and SF$\mathrm{P}-\mathrm{Mn}$ (II) resin polymer spheres and their corresponding carbon spheres is shown in Fig. 5. In a typical Stöber method, stable emulsion droplets are formed without any surfactant through hydrogen bonding interactions between water, methanol and 4,4'-dihydroxysalen Schiff base. Then, polymerization of $4,4^{\prime}$ dihydroxysalen and formaldehyde takes place inside the droplets by ammonia catalysis. During this process, $4,4^{\prime}$-dihydroxysalen, formaldehyde and ammonia react to form numerous condensation compounds such as hydroxymethyl substituted species that cross-link together and result in the formation of uniform colloidal SF polymer spheres. The ammonium cations not only accelerate the polymerization reaction between salen and formaldehyde in the emulsion, but also supply the positive charges distributing on the outer surface of polymer spheres that prevent the aggregation of the resin spheres. ${ }^{13}$

SEM images of the SF resin spheres before and after hydrothermal treatment (SF-MW and SF-MW $\mathrm{HT}_{\mathrm{HT}}$, respectively) are shown in Fig. 6a and b.
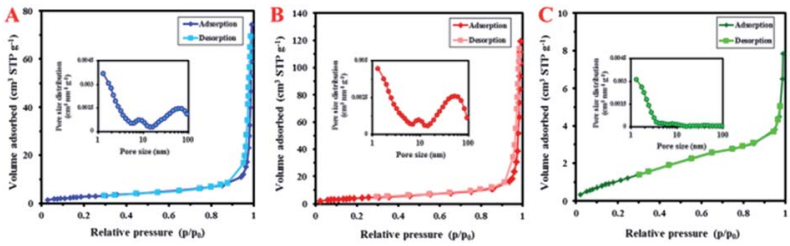

Fig. $7 \quad N_{2}$ adsorption-desorption isotherms of (A) SF-MW, (B) SF$\mathrm{MW}_{\mathrm{HT}}$, and (C) SF-M $\mathrm{M}_{\mathrm{HT}}$ samples. The insets show the corresponding BJH curves.
Table 1 The parameters of nitrogen adsorption of the SF resin polymers

\begin{tabular}{llll}
\hline Sample & $S_{\text {BET }}\left(\mathrm{m}^{2} \mathrm{~g}^{-1}\right)$ & $V_{\text {TP }}\left(\mathrm{cm}^{3} \mathrm{~g}^{-1}\right)$ & $D_{\mathrm{MP}}(\mathrm{nm})$ \\
\hline SF-MW & 10.88 & 0.11 & 41.21 \\
SF-MW $_{\text {HT }}$ & 15.78 & 0.18 & 46.72 \\
SF-M $_{\mathrm{HT}}$ & 4.08 & 0.01 & 11.74
\end{tabular}

It is clear that the colloidal spheres had irregular shapes before hydrothermal treatment (Fig. 6a) and they became more spherical with smooth surfaces and reduced roughness after hydrothermal processing (Fig. 6b). ${ }^{37}$ It was also found that the solvent has an impact on the size of the obtained spheres. From Fig. $6 \mathrm{c}$ it is clear that in the case of the SF- $\mathrm{M}_{\mathrm{HT}}$ colloidal spheres, although absolute methanol as a solvent leads to the production of smoother spherical particles, instead it results in an increase in their size.

(a)

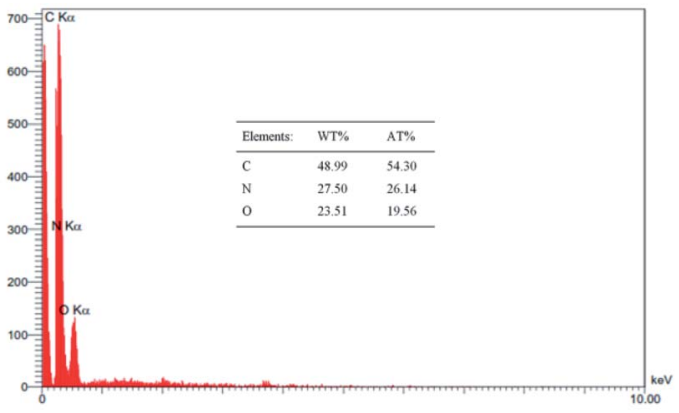

(b)

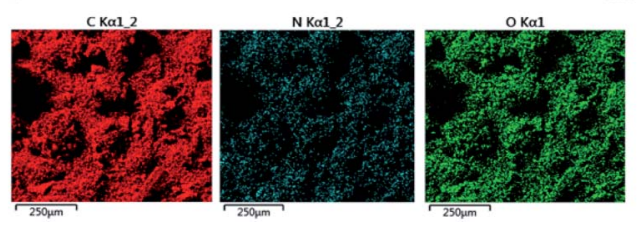

Fig. 8 (a) EDX analysis for the SF-MW $\mathrm{HT}_{\mathrm{H}}$ resin polymer spheres, and (b) the corresponding elemental mapping. The inset shows the elemental analysis data. 

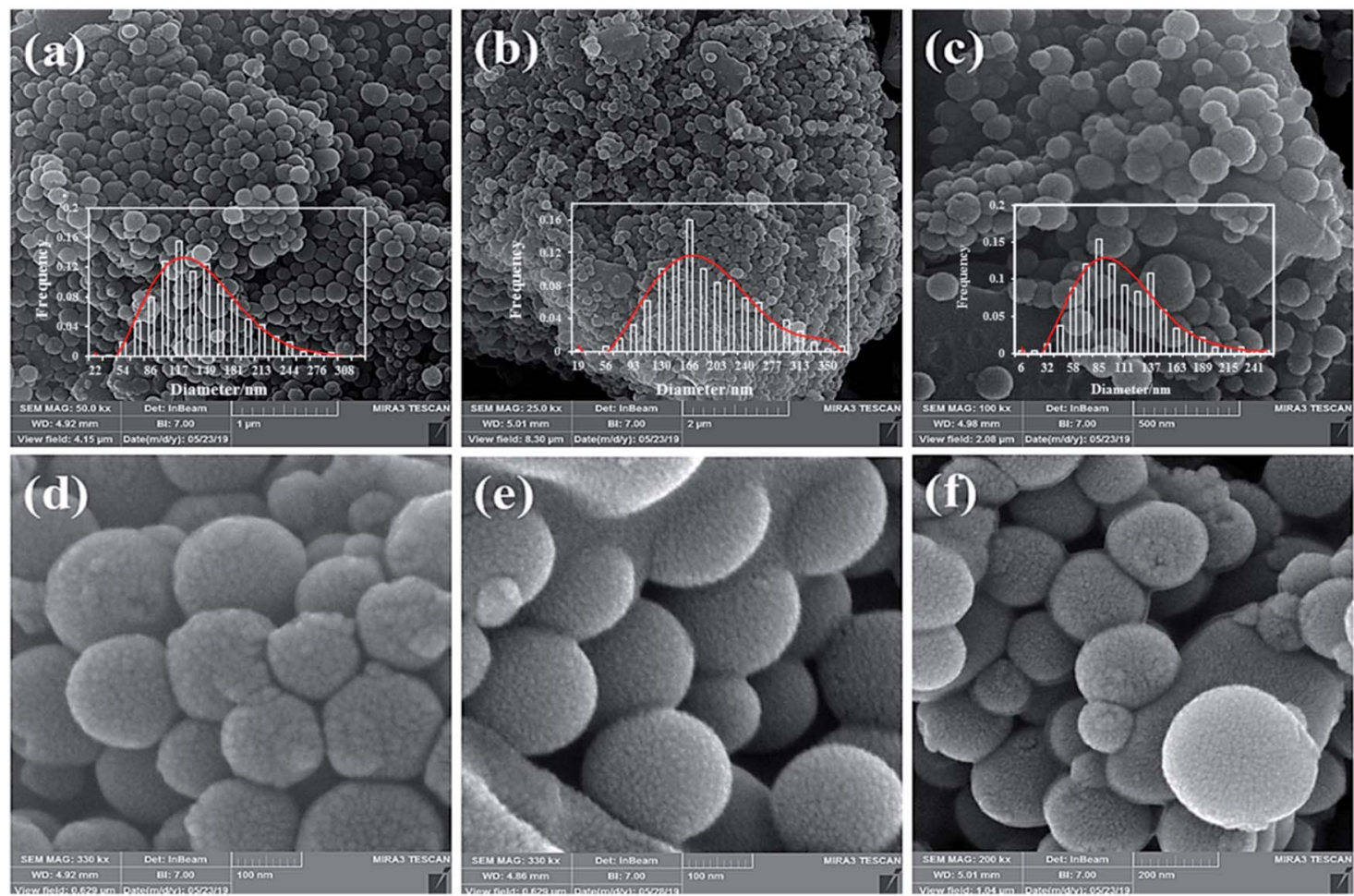

Fig. 9 SEM images of ( $a$ and d) SF-C, (b and e) SF-P-C, and ( $c$ and f) SF-P-CaMn. The insets show the corresponding particle size distribution curves.

$\mathrm{N}_{2}$ adsorption-desorption isotherms of the SF samples are shown in Fig. 7. This study provided valuable information on the specific surface area and porosity of the prepared resin polymers. The SF resin spheres before and after hydrothermal treatment showed a type IV $\mathrm{N}_{2}$-sorption isotherm with distinct hysteresis loops in the range of $p / p_{0}$ from 0.85 to 1.0 , indicative of porous adsorbents with pores in the range of 1.5-100 nm. The SF- $\mathrm{M}_{\mathrm{HT}}$ sample, on the other hand, showed a type II $\mathrm{N}_{2^{-}}$ sorption isotherm, characteristic of powders with diameters exceeding micropores. The corresponding Barrett-JoynerHalenda (BJH) curves (insets of Fig. 7), clearly indicate the presence of micro-, meso, and macropores in the SF-MW and


(i)
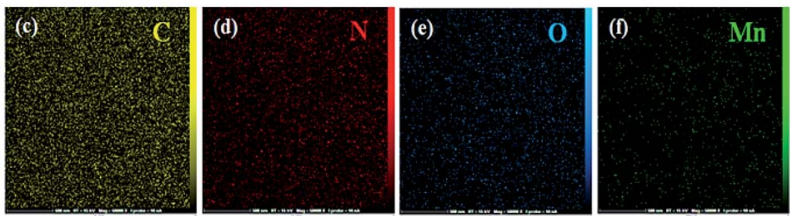

Fig. 11 (a) EDX spectrum of the SF-P-CaMn carbon spheres, and (b-f) the corresponding mapping images. The inset shows the elemental analysis data. 

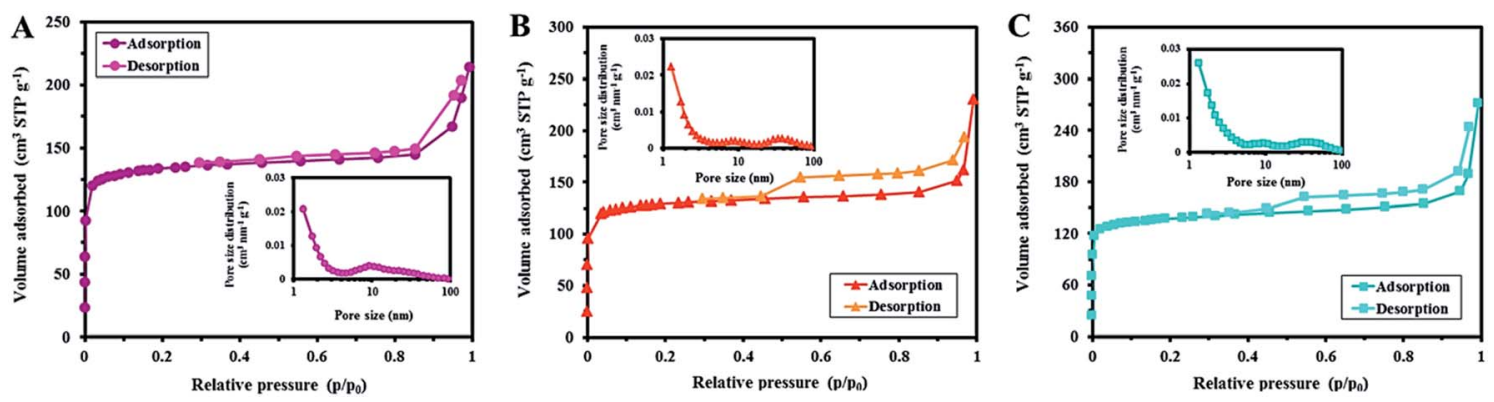

Fig. $12 \mathrm{~N}_{2}$ adsorption-desorption isotherms of (A) SF-C, (B) SF-P-C, and (C) SF-P-CaMn carbon spheres. The insets show the corresponding BJH curves.

Nitrogen content of the SF-MW $\mathrm{HT}_{\mathrm{HT}}$ resin polymer spheres was determined by energy dispersive X-ray (EDX) analysis (Fig. 8). As the inset of Fig. 8a shows, nitrogen content of the SF-MW sample was about 27.5 wt\%. EDX-mapping (Fig. 8b), on the other hand, revealed homogeneous distribution of the nitrogen atoms throughout the sample.

FT-IR spectrum of the SF-MW $\mathrm{HT}_{\mathrm{HT}}$ sample is shown in Fig. $2 \mathrm{~b}$ and exhibits the anticipated bands for $\nu_{\mathrm{O}-\mathrm{H}}, \nu_{\mathrm{C}=\mathrm{N}}$, and $\nu_{\mathrm{Ph}-\mathrm{O}}$ at 3420,1635 , and $1234 \mathrm{~cm}^{-1}$, respectively.

Fig. 9 shows the SEM images of the carbonization products SF-C, SF-P-C and SF-P-C@Mn. It is clearly seen that the obtained carbon spheres have regular spherical shapes with smooth surfaces.

After carbonization, however, some defects were observed on the surface of carbon particles as a result of shrinkage during the calcination process. ${ }^{18}$ It is noteworthy that the use of Pluronic $\mathrm{F} 127$ as a template resulted in an increase in particle size from about $140 \mathrm{~nm}$ for the SF-C to about $188 \mathrm{~nm}$ for the SF-P-C samples, respectively. The decrease in particle size to about $106 \mathrm{~nm}$ in the SF-P-C@Mn sample may be related to the presence of manganese species.

TEM imaging further demonstrated that the carbonization products were monodispersed and homogeneously spherical (Fig. 10).

To further characterize the obtained carbon spheres, EDX analysis was performed. The results for the SF-C and SF-P-C samples are shown in Fig. $\mathrm{S} 1$ and $\mathrm{S} 2, \dagger$ and clearly indicate homogeneous distribution of the $\mathrm{C}, \mathrm{N}$, and $\mathrm{O}$ atoms in their structure. EDX spectrum of the SF-P-C@Mn sample is shown in Fig. 11a and confirms the presence of manganese in the carbonized spheres.

Nitrogen and manganese content of the SF-P-C@Mn carbon spheres were found to be 35 and $0.18 \mathrm{wt} \%$, respectively (table

Table 2 Physico-chemical properties of the carbonized samples

\begin{tabular}{llll}
\hline Sample & $S_{\text {BET }}\left(\mathrm{m}^{2} \mathrm{~g}^{-1}\right)$ & $V_{\mathrm{TP}}\left(\mathrm{cm}^{3} \mathrm{~g}^{-1}\right)$ & $D_{\text {MP }}(\mathrm{nm})$ \\
\hline SF-C & 499 & 0.33 & 2.54 \\
SF-P-C & 516.83 & 0.35 & 2.78 \\
SF-P-C@Mn & 528.49 & 0.41 & 3.08
\end{tabular}

inset of Fig. 11a). Also, homogeneous distribution of the C, N, O and $\mathrm{Mn}$ constituents is evident from Fig. 11b-f.

Porosity of the carbonized samples was also studied. $\mathrm{N}_{2}$ adsorption-desorption isotherms of the carbonized samples are shown in Fig. 12. While the SF-C sample exhibited a type II isotherm, the other two showed a type IV isotherm with distinct hysteresis loops in the range of $p / p_{0}$ from 0.3 to 1.0 . Physicochemical properties of the carbonized samples are listed in Table 2. Clearly, specific surface area $\left(S_{\mathrm{BET}}\right)$, total pore volume $\left(V_{\mathrm{TP}}\right)$, and mean pore diameter $\left(D_{\mathrm{MP}}\right)$ of each sample was improved after calcination.

In the case of the SF-C sample, a significant improvement in the BET surface area from 15.78 to $499 \mathrm{~m}^{2} \mathrm{~g}^{-1}$ was observed which can be attributed to the loss of water, $\mathrm{NH}_{4}{ }^{+}$and other functional groups during the carbonization process. ${ }^{38}$

Total pore volume was also increased accordingly from 0.18 to $0.33 \mathrm{~cm}^{3} \mathrm{~g}^{-1}$. In the case of the SF-P-C and SF-P-C@Mn samples, on the other hand, removal of the template and complex formation effects may contribute to further enhance the above mentioned properties. ${ }^{11}$ The corresponding BJH curves (insets of Fig. 12), clearly indicate the presence of microand mesopores in the structure of SF, and micro-, meso-, and macropores in the SF-P and SF-P-C@Mn carbon spheres.

XRD patterns for the SF-C, SF-P-C and SF-P-C@Mn samples are shown in Fig. 13. As shown, the obtained carbon spheres are

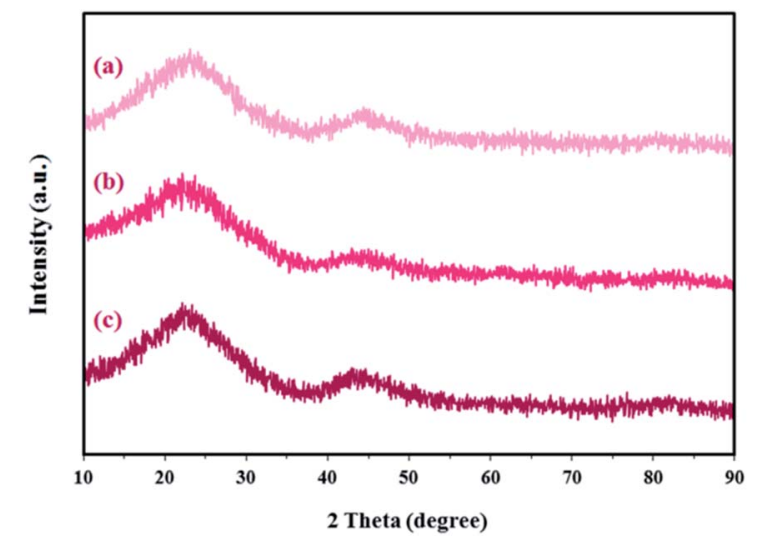

Fig. 13 X-ray diffraction patterns for the (a) SF-C, (b) SF-P-C, and (c) SF-P-C@Mn samples. 

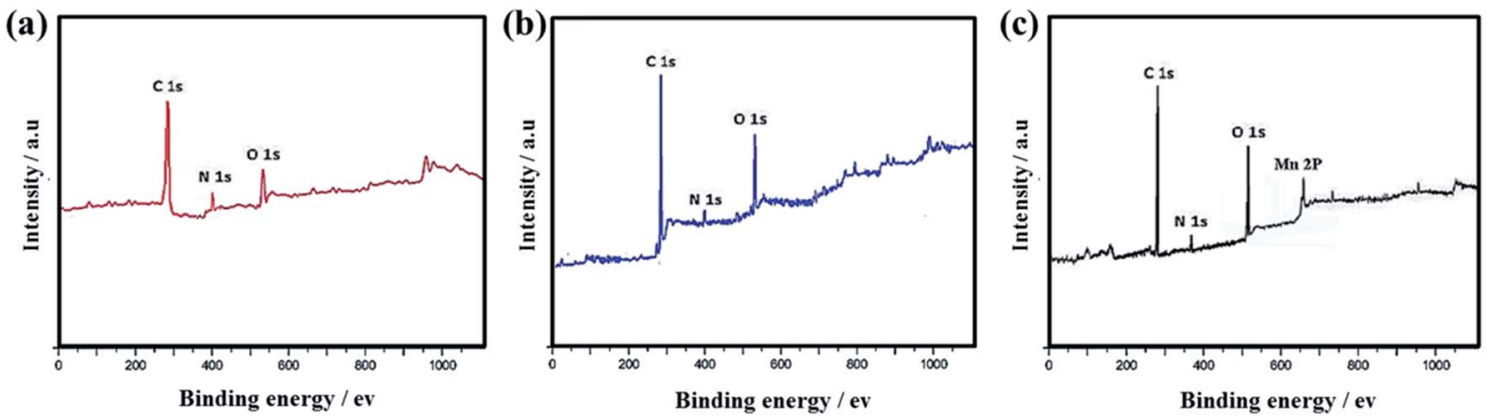

Fig. 14 XPS survey spectra of (a) SF-C, (b) SF-P-C and (c) SF-P-CaMn carbon spheres.

amorphous. It can be concluded that the use of Pluronic F127 or complexation with $\mathrm{Mn}$ (II) does not lead to crystallinity of the obtained carbon spheres. Broad diffraction peaks at 2 theta angles about $23^{\circ}$, can be ascribed to the (002) plane of amorphous carbon. ${ }^{22}$

Fig. 2c and d show the FT-IR spectra of the SF-P-C and SF-PC@Mn samples. Although most of the characteristic peaks of the parent scaffolds were present, band intensities were decreased to some extent. Also, in the FT-IR spectrum of SF-PC@Mn (Fig. 2d), a new peak was appeared at $608 \mathrm{~cm}^{-1}$ which can be attributed to the $\mathrm{Mn}-\mathrm{O}$ vibrations. ${ }^{39}$

XPS spectra in Fig. 14a-c show that $\mathrm{C} 1 \mathrm{~s}, \mathrm{~N}$ 1s and $\mathrm{O} 1 \mathrm{~s}$ characteristic peaks exist in SF-C, SF-P-C and SF-P-C@Mn, and Mn 2p is present in SF-P-C@Mn, which is in good agreement with EDX-mapping results.

\section{Electrocatalytic performance}

Eventually, catalytic activity of the SF-P-C@Mn sample was evaluated in the oxygen reduction reaction. LSVs of the GCE and MGCE in $0.1 \mathrm{M} \mathrm{KOH}$ are compared in Fig. 15. As shown, the ORR was carried out very late and very slowly at the surface of GCE, which indicates the lack of electrocatalytic performance.

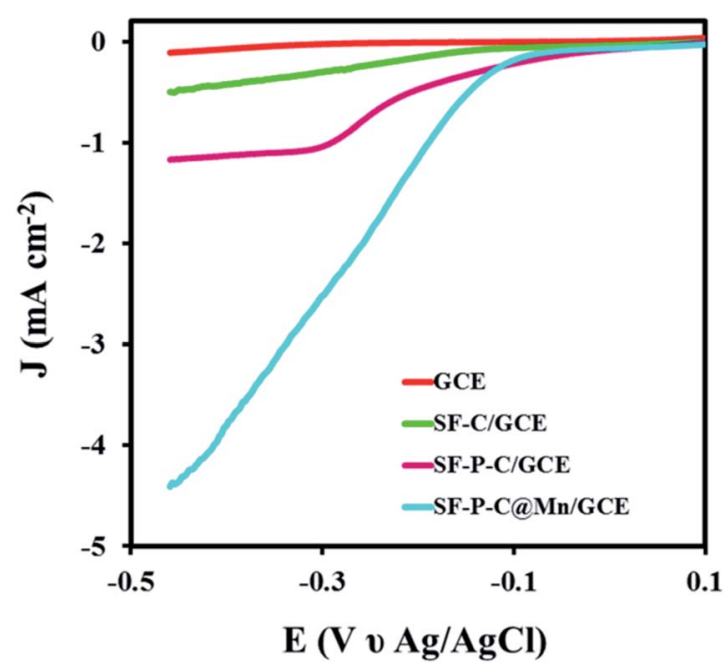

Fig. 15 LSVs of GCE, SF-C/GCE, SF-P-C/GCE, and SF-P-CaMn/GCE in $0.1 \mathrm{M} \mathrm{KOH}$ at a scan rate of $0.05 \mathrm{mV} \mathrm{s}^{-1}$.
In contrast, the ORR for SF-C/GCE, SF-P-C/GCE, and SF-PC@Mn/GCE was performed at $-0.24,-0.18$, and $-0.11 \mathrm{~V}$, respectively. The results show that SF-P-C@Mn/GCE has the higher potential for the reaction.

The Tafel slope and the exchange current density $\left(J_{0}\right)$ (Fig. 16) were calculated according to the following equation:

$$
E=R T / \alpha n F \ln J_{0}-R T / \alpha n F \ln J
$$

where $\alpha, F, R, J_{0}, J$, and $T$ are the transfer coefficient, the Faraday constant, the gas constant, exchange current density, and the absolute temperature, respectively. As listed in Table 3, the Tafel slope and the current density of the modified electrode with SFP-C@Mn nanocomposite is higher than the electrode modified with SF-C, SF-P-C.

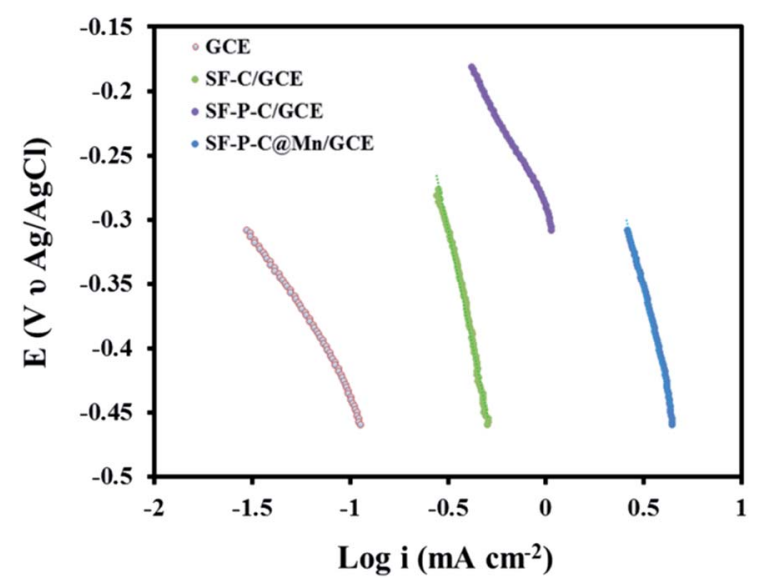

Fig. 16 Tafel plot of GCE, SF-C/GCE, SF-P-C/GCE, and SF-P-C@Mn/ $\mathrm{GCE}$ in $0.1 \mathrm{M} \mathrm{KOH}$ at a scan rate of $5 \mathrm{mV} \mathrm{s}^{-1}$.

Table 3 The Tafel slope and current density of difference electrodes

\begin{tabular}{lcl}
\hline Electrode & $\begin{array}{l}\text { Tafel slope } \\
\left(\mathrm{mV} \mathrm{dec}^{-1}\right)\end{array}$ & $\begin{array}{l}\text { Current density } \\
\left(\mathrm{mA} \mathrm{cm}^{-2}\right)\end{array}$ \\
\hline GCE & 41.23 & 20.51 \\
SF-C/GCE & 73.41 & 33.29 \\
SF-P-C/GCE & 101.38 & 45.83 \\
SF-P-C@Mn/GCE & 138.12 & 70.32
\end{tabular}




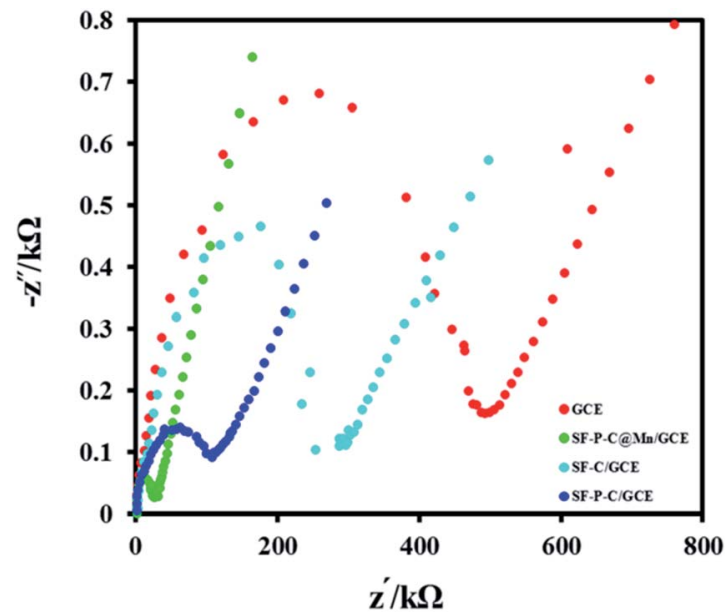

Fig. 17 Nyquist curves obtained from electrochemical impedance spectroscopy measurements in $0.1 \mathrm{M} \mathrm{KOH}$.

In order to examine the resistance to charge transfer at the electrode surface during the ORR process, electrochemical impedance spectroscopy in $0.1 \mathrm{M} \mathrm{KOH}$ with a potential of $-0.11 \mathrm{~V}$ within a frequency range of $10.0 \mathrm{kHz}$ to $10 \mathrm{MHz}$ with an amplitude of $10 \mathrm{mV}$ AC was used. The corresponding Nyquist curves are shown in Fig. 17. As shown, resistance to charge transfer $\left(R_{\mathrm{CT}}\right)$ of the SF-P$\mathrm{C} @ \mathrm{Mn} / \mathrm{GCE}$ is much lower than the SF-P-C/GCE, SF-C/GCE, and GCE which indicates high electrocatalytic performance of the modified electrode in the ORR process.

In order to determine the stability of the modified electrode in the ORR process, chronoamperometry technique was used. For this purpose, the SF-P-C@Mn/GCE was placed in $0.1 \mathrm{M} \mathrm{KOH}$ at a constant potential of $-0.11 \mathrm{~V}$ for $3600 \mathrm{~s}$, and the chronoamperogram was recorded (Fig. 18). Clearly, a constant current was obtained over the specified time period, which indicates durability and stability of the modified electrode.

Also, the stability of the SF-P-C@Mn/GCE was checked in the ORR process once a week consecutively for 4 weeks (with chronoamperometry technique). The results indicate that the desired SF-P-C@Mn/GCE surface has long-term stability and in

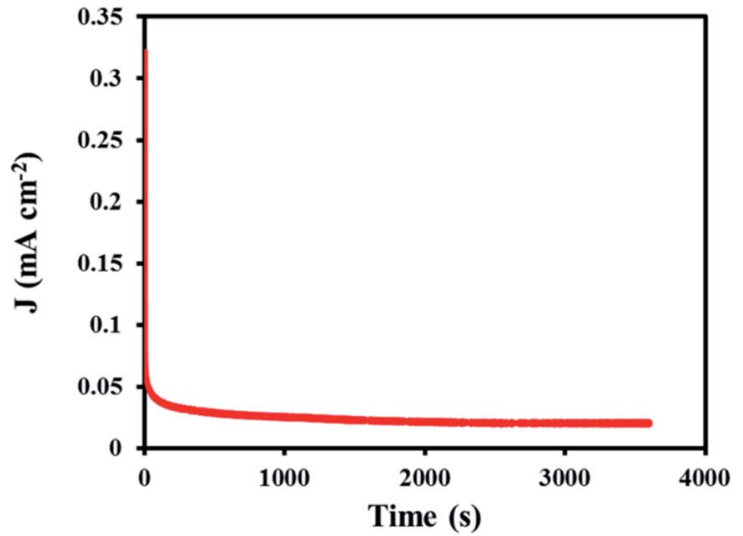

Fig. 18 The recorded chronoamperogram for SF-P-C@Mn/GCE in $0.1 \mathrm{M} \mathrm{KOH}$ during $3600 \mathrm{~s}$. the $4^{\text {th }}$ week, only a $4.89 \%$ signal decrease was observed compared with the first week.

\section{Conclusion}

An extension of the Stöber method was used to prepare monodispersed salen-formaldehyde resin polymer- and carbon spheres with high nitrogen content. The results showed that the solvent system used has an impact on the properties of the obtained SF resin spheres. Pluronic F127 as a template and porogenic agent significantly improved the quality of the obtained resin spheres and their descendant carbon analogues. $\mathrm{Mn}$ (II)-coordinated polymer spheres of the SF resin were also synthesized.

The SF-P-Mn(II) complex was then converted to SF-P-C@Mn spheres after carbonization under an inert atmosphere. Interesting physico-chemical properties of the obtained SF-P-C@Mn carbon particles, including a BET surface area of $528 \mathrm{~m}^{2} \mathrm{~g}^{-1}$ and a high nitrogen content of about $35 \mathrm{wt} \%$, provided a suitable basis for modification of a glassy carbon electrode to be used in the oxygen reduction reaction. SF-P-C@Mn/GCE was successfully used as an electrocatalyst in the ORR process. The improved electrocatalytic activity may be attributed to the presence of Mn nanoparticles in the SF-P-C@Mn structure as was evident from the FT-IR and TEM analyses.

\section{Conflicts of interest}

There are no conflicts to declare.

\section{Acknowledgements}

We gratefully acknowledge the support of the Research Council of University of Guilan.

\section{References}

1 A. H. Lu, W. Schmidt, N. Matoussevitch, H. Bonnemann, B. Spliethoff, B. Tesche, E. Bill, W. Kiefer and F. Schuth, Angew. Chem., Int. Ed., 2004, 43, 4303-4306.

2 A. H. Lu, T. Sun, W. C. Li, Q. Sun, F. Han, D. H. Liu and Y. Guo, Angew. Chem., Int. Ed., 2011, 50, 11765-11768.

3 J. Wang, H. Liu, J. Diao, X. Gu, H. Wang, J. Rong, B. Zong and D. S. Su, J. Mater. Chem. A, 2015, 3, 2305-2313.

4 T. Yang, R. Zhou, D. W. Wang, S. P. Jiang, Y. Yamauchi, S. Z. Qiao, M. J. Monteiro and J. Liu, Chem. Commun., 2015, 51, 2518-2521.

5 Y. Fang, G. Zheng, J. Yang, H. Tang, Y. Zhang, B. Kong, Y. Lv, C. Xu, A. M. Asiri, J. Zi and F. Zhang, Angew. Chem., Int. Ed., 2014, 53, 5366-5370.

6 L. Zhong, C. Anand, K. Lakhi, G. Lawrence and A. Vinu, Sci. Rep., 2015, 5, 12901-12908.

7 W. Sun, M. Chen, S. Zhou and L. Wu, Langmuir, 2014, 30, 12011-12017.

8 J. Zhu, L. Liao, X. Bian, J. Kong, P. Yang and B. Liu, Small, 2012, 8, 2715-2720. 
9 W. M. Zhang, J. S. Hu, Y. G. Guo, S. F. Zheng, L. S. Zhong, W. G. Song and L. J. Wan, Adv. Mater., 2008, 20, 1160-1165.

10 L. F. Chen, X. D. Zhang, H. W. Liang, M. Kong, Q. F. Guan, P. Chen, Z. Y. Wu and S. H. Yu, ACS Nano, 2012, 6, 70927102.

11 P. Zhang, Z. A. Qiao and S. Dai, Chem. Commun., 2015, 51, 9246-9256.

12 W. Stöber, A. Fink and E. Bohn, J. Colloid Interface Sci., 1968, 26, 62-69.

13 J. Liu, S. Z. Qiao, H. Liu, J. Chen, A. Orpe, D. Zhao and G. Q. Lu, Angew. Chem., Int. Ed., 2011, 50, 5947-5951.

14 J. Xu, A. Wang and T. Zhang, Carbon, 2012, 50, 1807-1816. 15 A. H. Moreno, A. Arenillas, E. G. Calvo, J. M. Bermúdez and J. A. Menéndez, J. Anal. Appl. Pyrolysis, 2013, 100, 111-116.

16 X. Sun and Y. Li, Angew. Chem., Int. Ed., 2004, 43, 597-601. 17 J. Zhao, C. L. Li and R. Liu, Nanoscale, 2018, 10, 5882-5887. 18 M. Liu, C. Cai, J. Li, J. Zhao, W. Teng and R. Liu, J. Colloid Interface Sci., 2018, 528, 1-9.

19 R. Liu, F. Qu, Y. Guo, N. Yao and R. D. Priestley, Chem. Commun., 2013, 50, 478-480.

20 G. Wu, Z. Chen, F. H. Garzon and P. Zelenay, ECS Trans., 2008, 16, 159-170.

21 X. Zhu, S. Wang, W. Huang, Y. Tian and X. Wang, Carbon, 2016, 105, 521-528.

22 J. C. Song, Z. Y. Lu and Z. Y. Sun, J. Colloid Interface Sci., 2014, 431, 132-138.

23 J. Liang, Y. Jiao, M. Jaroniec and S. Z. Qiao, Angew. Chem., Int. Ed., 2012, 51, 11496-11500.

24 Y. Jiao, Y. Zheng, M. Jaroniec and S. Z. Qiao, J. Am. Chem. Soc., 2014, 136, 4394-4403.
25 X. Liu, C. Manzur, N. Novoa, S. Celedón, D. Carrillo and J. R. Hamon, Coord. Chem. Rev., 2018, 357, 144-172.

26 B. Huang, Y. Wang, S. Zhan and J. Ye, Appl. Surf. Sci., 2017, 396, 121-128.

27 Z. Wang, Y. Qin, C. Wang, L. Sun, X. Lu and X. Lu, Appl. Surf. Sci., 2012, 258, 2017-2021.

28 J. Ren, H. Xuan, W. Dai, Y. Zhu and L. Ge, Appl. Surf. Sci., 2018, 448, 609-617.

29 J. Tedim, S. Patrício, R. Bessada, R. Morais, C. Sousa, M. B. Marques and C. Freire, Eur. J. Inorg. Chem., 2006, 17, 3425-3433.

30 L. Martínez-Rodríguez, N. A. Bandeira, C. Bo and A. W. Kleij, Chem.-Eur. J., 2015, 21, 7144-7150.

31 Y. Xu, J. Meng, L. Meng, Y. Dong, Y. Cheng and C. Zhu, Chem.-Eur. J., 2010, 16, 12898-12903.

32 J. Wang, L. Huang, M. Xue, L. Liu, Y. Wang, L. Gao, J. Zhu and Z. Zou, Appl. Surf. Sci., 2008, 254, 5329-5335.

33 A. Erxleben, Inorg. Chim. Acta, 2018, 472, 40-57.

34 H. Tian, J. Liu, K. O'Donnell, T. Liu, X. Liu, Z. Yan, S. Liu and M. Jaroniec, J. Colloid Interface Sci., 2016, 476, 55-61.

35 T. Yang, J. Liu, R. Zhou, Z. Chen, H. Xu, S. Z. Qiao and M. J. Monteiro, J. Mater. Chem. A, 2014, 2, 18139-18146.

36 D. Dey, G. Kaur, M. Patra, A. R. Choudhury, N. Kole and B. Biswas, Inorg. Chim. Acta, 2014, 421, 335-341.

37 C. Liu, J. Wang, J. Li, X. Hu, P. Lin, J. Shen, X. Sun, W. Han and L. Wang, J. Mater. Chem. A, 2016, 4, 11916-11923.

38 S. Ren, M. Wang, C. Jia, C. Hao and X. Wang, Energy Technol., 2013, 1, 332-337.

39 A. Bayoudh, N. Etteyeb and F. Sediri, American Journal of Nanosciences, 2016, 2, 1-7. 